\title{
On the Optimal Object-Oriented Program Re-modularization
}

\author{
Saeed Parsa and Omid Bushehrian \\ Faculty of Computer Engineering, Iran University of Science and Technology \\ \{parsa, bushehrian\} aiust.ac.ir
}

\begin{abstract}
In this paper a new criterion for automatic re-modularization of object-oriented programs is presented. The aim of re-modularization here is to determine a distributed execution of a program over a dedicated network of computers with the shortest execution time. To achieve this, a criterion to quantitatively evaluate performance of a re-modularized program is presented as a function. This function is automatically constructed while traversing the program call flow graph once before the search for the optimal remodularization of the program and considers both synchronous and asynchronous types for each call within the call flow graph.
\end{abstract}

\section{Introduction}

With the increasing popularity of using clusters and network of low cost computers in solving computationally intensive problems, there is a great demand for system and application software that can provide transparent and efficient utilization of the multiple machines in a distributed system [2][3][5]. There are a number of such application softwares including middle-wares and utility libraries which support parallel and distributed programming over a network of machines. A distributed program written using these middle-wares comprises a number of modules or distributed parts communicating by means of message passing or asynchronous method calls.

Our aim has been to develop automatic techniques to obtain maximum execution concurrency among distributed parts or modules of a program. To reach this end, the main difficulty is to determine theses distributed parts, or equivalently, the architecture of a distributed program code. The architecture of a program can be reconstructed using software reverse engineering and re-modularization techniques $[1][4]$.

\section{The Optimal Re-modularization of a Program}

Each clustering of a program call graph, which is a modularization of that program, represents a subset of program method calls, named remote-call set, to be converted to remote asynchronous calls. For instance consider the modularized call graph of four classes in Figure 1. This modularization corresponds to remote-call set $\{\mathrm{c} 1, \mathrm{c} 2, \mathrm{c} 4\}$. 


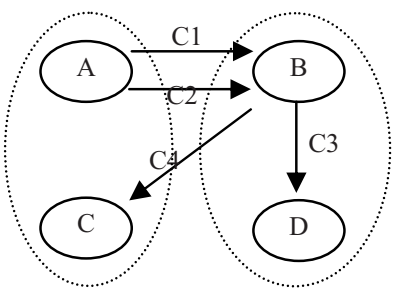

Fig. 1. Re-modularization of a program call graph

\section{Performance Estimation of a Re-modularized Program}

In order to evaluate a re-modularized program performance, the remote-call set corresponding to that re-modularization is obtained and evaluated by applying a function called Estimated Execution Time (EET). For a given remote-call set $r$, $E \mathrm{~T}_{1}(\mathrm{r})$ calculates a value which is an estimation of the amount of execution time of method call $I$ with respect to $r$. Each EET formula is generated from the program call flow graph (CFG). CFG shows the flow of method calls among program classes. Each node in this graph represents a method body in an abstract way by means of a sequence of symbols. Each symbol in this sequence indicates one of these concepts: a method invocation, a synchronization point between caller and callee methods or an ordinary program instruction which are denoted by $\mathrm{I}_{\mathrm{i}}, \mathrm{S}_{\mathrm{i}}$ and $\mathrm{W}_{\mathrm{i}}$ respectively. Symbol $\mathrm{S}_{\mathrm{i}}$ indicates the first program location which is data dependent to a method invocation $\mathrm{I}_{\mathrm{i}}$ in the CFG node sequence. Symbol $\mathrm{W}_{\mathrm{i}}$ represents any collection of ordinary program statements with estimated execution time i. Below in Figure 2 is a sample Java code and its corresponding CFG. EET function for a program is generated automatically by traversing the program CFG. Since each method invocation $\mathrm{I}_{\mathrm{i}}$ in the program CFG may be executed either synchronously or asynchronously, depending on the specified modularization of the program classes, the EET function includes time estimation for both synchronous and asynchronous execution types for each invocation $\mathrm{I}_{\mathrm{i}}$. For instance the EET function for $\mathrm{CFG}$ in Figure 2 is generated as follows:

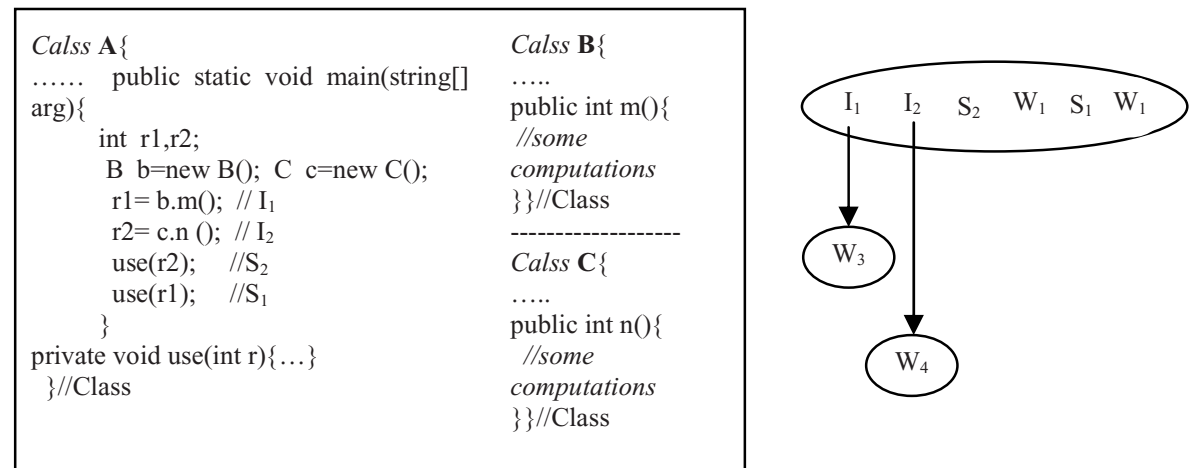

Fig. 2. A sample program including three classes and its CFG 
$\operatorname{EET}_{\text {main }}(\mathrm{r})=\mathrm{a}_{1} * \operatorname{EET}_{\mathrm{I} 1}(\mathrm{r})+\mathrm{a}_{2} * \operatorname{EET}_{\mathrm{I} 2}(\mathrm{r})+\left(1-\mathrm{a}_{2}\right) * \mathrm{~T}\left(\mathrm{~S}_{2}\right)+\mathrm{W}_{1}+\left(1-\mathrm{a}_{1}\right) * \mathrm{~T}\left(\mathrm{~S}_{1}\right)$

$+\mathrm{W}_{1} \quad \mathrm{EET}_{\mathrm{I} 1}(\mathrm{r})=\mathrm{W}_{3}, \mathrm{EET}_{\mathrm{I} 2}(\mathrm{r})=\mathrm{W}_{4}$,

In this relation, depending on the execution type of invocations $I_{1}$ and $I_{2}$, asynchronous or synchronous, coefficients $\mathrm{a}_{1}$ and $\mathrm{a}_{2}$ are set to 0 or 1 respectively. $\mathrm{S}_{1}$ and $S_{2}$ are synchronization points of calls $I_{1}$ and $I_{2}$ respectively and $T\left(S_{i}\right)$ indicates the amount of time that should be elapsed at synchronization point $S_{i}$ until invocation $I_{i}$ is completed.

The general form of an EET relation for a program is as follows:

$$
\operatorname{EET}_{\mathrm{m}}(\mathrm{r})=\sum \mathrm{w}_{\mathrm{i}}+\sum \mathrm{a}_{\mathrm{i}} * \operatorname{EET}_{\mathrm{Ii}}(\mathrm{r})+\sum\left(1-\mathrm{a}_{\mathrm{i}}\right) * \mathrm{~T}\left(\mathrm{~S}_{\mathrm{i}}\right)
$$

In the above formula coefficients $\mathrm{a}_{\mathrm{i}}$ are determined by remote-call set $r$ as follows:

$$
\mathrm{a}_{\mathrm{i}}= \begin{cases}1 & : \mathrm{I}_{\mathrm{i}} \notin \mathrm{r} \\ 0 & : \mathrm{I}_{\mathrm{i}} \in \mathrm{r}\end{cases}
$$

As described above, $\mathrm{T}\left(\mathrm{S}_{\mathrm{i}}\right)$ is the amount of time that should be elapsed at synchronization point $S_{i}$ until invocation $I_{i}$ is completed. $T\left(S_{i}\right)$ is calculated by the following relation:

$$
\mathrm{T}\left(\mathrm{S}_{\mathrm{i}}\right)=\max \left(\left(\operatorname{EET}_{\mathrm{Ii}}(\mathrm{r})+\mathrm{O}_{\mathrm{i}}\right)-\mathrm{t}_{\mathrm{i}}, 0\right)
$$

Where, $t_{i}$ is estimated execution time of the program fragment between symbols $\mathrm{I}_{\mathrm{i}}$ and $\mathrm{S}_{\mathrm{i}}$. Since each asynchronous method invocation $\mathrm{I}_{\mathrm{i}}$ imposes a communication overhead on the overall program execution time, this overhead which is denoted by $O_{i}$, is added to the estimated execution time of $\mathrm{I}_{\mathrm{i}}$. Since it is assumed that CFG is cycle free, $\operatorname{EET}_{m}(r)$ can be solved by recursively replacing EET terms until $\mathrm{EET}_{\mathrm{m}}(\mathrm{r})$ contains only $\mathrm{a}_{\mathrm{i}}$ coefficients, $\mathrm{W}_{\mathrm{i}}$ terms, $\mathrm{O}_{\mathrm{i}}$ terms and $\max$ operators.

\section{Conclusions}

The main difficulty in obtaining a distributed execution of a program with minimum execution time is to find the smallest set of program invocations to be converted to remote asynchronous invocations. Program re-modularization can be applied as an approach to reach this end. Program re-modularization is used to reconstruct program architecture with respect to one ore more quality constraints such as performance or maintainability. In this paper a new criterion for performance driven remodularization of a program has been proposed. This criterion is used to quantitatively estimate the performance of a re-modularized program with a function which is generated automatically from the program call flow graph (CFG). This function includes time estimations for both asynchronous and synchronous execution types of each method in the program call flow graph. 


\section{References}

1. Berndt Bellay, Harald Gall, "Reverse Engineering to Recover and Describe a Systems Architecture", Development and Evolution of Software Architectures for Product Families, Lecture Notes in Computer Science(1998), Volume 1429.

2. Bushehrian Omid, Parsa Saeed, "Formal Description of a Runtime Infrastructure for Automatic Distribution of Programs", The $21^{\text {th }}$ International Symposium on Computer and Information Sciences, Lecture Notes in Computer Science(2006), Vol. 4263.

3. Jameela Al-Jaroodi, Nader Mahamad, Hong Jiang, David Swanson, "JOPI: a Java Object Passing Interface", Concurrency Computat. : Pract. Exper. (2005); 17:775-795

4. Parsa S., Bushehrian O., "The Design and Implementation of a Tool for Automatic Software Modularization”, Journal of Supercomputing, Volume 32, Issue 1, April (2005).

5. Parsa Saeed, Khalilpour Vahid, "Automatic Distribution of Sequential Code Using JavaSymphony Middleware”, SOFSEM06, Lecture Notes in Computer Science(2006), Vol. 3831 , 